\title{
Design and studies of novel polyoxysterol-based porphyrin conjugates
}

\author{
Halina A. Zhylitskaya ${ }^{\mathrm{a}, \mathrm{b}}$, Vladimir N. Zhabinskii ${ }^{\mathrm{a}}$, Raisa P. Litvinovskaya ${ }^{\mathrm{a}}$, Raffaella Lettieri ${ }^{\mathrm{b}}$, \\ Donato Monti ${ }^{\mathrm{b}}$, Mariano Venanzi ${ }^{\mathrm{b}}$, Vladimir A. Khripach ${ }^{\mathrm{a}, *}$, Pavel Drašar $^{\mathrm{c}}$ \\ ${ }^{a}$ Institute of Bioorganic Chemistry, National Academy of Sciences of Belarus, Kuprevich str., 5/2, 220141 Minsk, Belarus \\ ${ }^{\mathrm{b}}$ Dipartimento di Scienze e Tecnologie Chimiche, Univ. Roma "Tor Vergata", I-00133 Rome, Italy \\ 'Institute of Chemical Technology, Technická 5, CZ-166 28 Praha 6, Czech Republic
}

\section{A R T I C L E I N F O}

\section{Article history:}

Received 4 May 2012

Received in revised form 28 June 2012

Accepted 3 July 2012

Available online 21 July 2012

\section{Keywords:}

Brassinosteroids

Ecdysteroids

Porphyrins

Conjugates

J-aggregates

\begin{abstract}
A B S T R A C T
New types of steroid-porphyrin conjugates derived from 20-hydroxyecdysone (20E) and 24-epibrassinolide ( $\mathrm{EBl})$ were synthesized. An exceptional regioselectivity in the reaction of both steroids with porphyrin boronic acids was found to give side-chain-conjugated boronic esters as sole products. UV-Vis-, fluorescence and NMR spectroscopy yielded similar data for all the studied compounds confirming the solvent driven supramolecular assembly with formation of J-aggregates. CD measurements of water diluted solutions showed a clear difference between $20 \mathrm{E}$ and EBI conjugates. The latter showed a strong supramolecular chirality, whereas $20 \mathrm{E}$ J-aggregates did not.
\end{abstract}

(c) 2012 Elsevier Inc. All rights reserved.

\section{Introduction}

Steroid frame has been quite widely utilized as a building block for supramolecular materials [1]. It is large, rigid, chiral, and thus suitable for creating of extended architectures with well-defined conformations, capable in principle to enantiodiscrimination [2]. Among numerous supramolecular systems based on the use of steroids, those derived from conjugation with porphyrins should be mentioned in the first place. They are used as photosensitizers for photodynamic therapy [3], in saccharide sensing [4-9], and anion binding [10]. Nearly all studies have been limited so far to bileacid based structures [11-14], with a few exceptions of porphyrin conjugates of estrogens [15-17], androgens [18,19] and cholesterol [20].

Oxygenated sterols form a large group of naturally occurring steroids widely spread in plants and animals and playing an important role as physiologically active substances. Brassinosteroids (BS, hormones of plants) [21] and ecdysteroids (ES, hormones of arthropods) [22] belong to the most remarkable members of the series. Representatives of these hormones are promising potential medications and active ingredients for new ecologically safe

Abbreviations: 20E, 20-hydroxyecdysone; BS, brassinosteroids; DDQ 2,3dichloro-5,6-dicyanobenzoquinone; DMA, dimethylacetamide; EBl, 24-epibrassinolide; ES, ecdysteroids.

* Corresponding author. Tel./fax: +375 172678647.

E-mail address: khripach@iboch.bas-net.by (V.A. Khripach). agrochemicals. BS and ES share similar structural elements involving a diol function in the cycle $A$ and a polyoxygenated side chain. Specific molecular structure of these compounds possessing a fixed rigid stereochemistry along with a number of chiral centres and functional groups makes them possible to be used as chiral ligands and potential sensor components for selective recognition of complex natural compounds and their synthetic analogs in instrumental analysis.

For a number of years, we have been interested in various aspects of pyrrole-steroid conjugates $[5,13,23-30]$ having in mind their expected value as new fluorescent probes for biochemical studies and immunoanalysis [31], and as possible sensing elements in special analytical devices [32] for recognizing the corresponding hormones perception. The main purposes of the present work were (1) to synthesize new steroid-porphyrin conjugates based on oxygenated sterols belonging to BS and ES and (2) to investigate physicochemical properties of the newly prepared compounds.

\section{Experimental}

\subsection{General}

Melting points were determined on a Boetius hot stage. Mass spectra were recorded on a $\mathrm{LCQ}^{\mathrm{TM}}$ ion-trap MS instrument (Thermo Electron, D-Dreich) (EI), $\mathrm{N}_{2}$ served as sheath and auxiliary gas, and He (purity $>99.9990 \%$, Messer-Griesheim, D-Krefeld) as collision gas. Mass spectra of conjugates were obtained on Bruker-Daltonics 
Biflex IV MALDI-TOF mass spectrometer equipped with standard nitrogen laser $(337 \mathrm{~nm}$ ) in a reflector mode. For evaluation of MALDI-TOF MS spectra mMass software was used [33]. High resolution ESIMS were obtained on Thermo Fischer Scientific LTQ Orbitrap Velos. Analysis was carried by positive and negative ionization. ${ }^{1} \mathrm{H}$ NMR and ${ }^{13} \mathrm{C}$ NMR spectra were recorded as solutions in $\mathrm{CD}_{3} \mathrm{OD}$, $\mathrm{C}_{5} \mathrm{D}_{5} \mathrm{~N}$ or $\mathrm{CDCl}_{3}$ on a Bruker AVANCE-500 spectrometer or on Varian 300 spectrometer. Chemical shift values are given in $\delta$ (ppm) relative to the residual solvent peaks: $\delta_{\mathrm{H}} 3.31$ for $\mathrm{CD}_{3} \mathrm{OD} ; \delta_{\mathrm{H}} 7.58$ and $\delta_{\mathrm{C}} 135.91$ for $\mathrm{C}_{5} \mathrm{D}_{5} \mathrm{~N} ; \delta_{\mathrm{H}} 7.26$ and $\delta_{\mathrm{C}} 77.00$ for $\mathrm{CDCl}_{3} ; \delta_{\mathrm{H}} 2.50$ for DMSO- $\mathrm{d}_{6}$ as references, and coupling constants are reported in $\mathrm{Hz}$.

Column chromatography and TLC were carried out using Merck silica gel 60: 70-230 mesh and precoated silica gel $60 \mathrm{~F}_{254}$ plates, respectively. Spots on TLC were visualized under UV light and by spraying with anisaldehyde- $\mathrm{H}_{2} \mathrm{SO}_{4}$ or ammonium cerium (IV) sulfate- $\mathrm{H}_{2} \mathrm{SO}_{4}$ reagents followed by heating. Solvents were dried and freshly distilled according to common practice.

UV spectra were measured on UV-Visible Spectrophotometer Varian Cary 1E in DMSO and DMA solutions at room temperature. Fluorescence spectra were collected on Jasco 7850, and FluoroMax2 Spectrofluorophotometers in $30 \mathrm{nM}$ solutions in DMSO and DMA. CD spectra were measured on JASCO J-600, equipped with a thermostated cell holder and purged with ultra-pure nitrogen gas, in DMA and DMSO solution (1 mkM) and in DMA- $\mathrm{H}_{2} \mathrm{O}(20-$ $60 \% \mathrm{v} / \mathrm{v}$ water content), DMSO- $\mathrm{H}_{2} \mathrm{O}(20-60 \% \mathrm{v} / \mathrm{v}$ water content) mixtures in $5 \mathrm{~h}$ and in 1 day after water addition.

Solvent-induced aggregation was studied by examining UV and fluorescence spectra changes against water content and time. $10-80 \% \mathrm{v} / \mathrm{v} \mathrm{H}_{2} \mathrm{O}$-DMSO and $\mathrm{H}_{2} \mathrm{O}$-DMA solutions were measured directly after water addition and in $1 \mathrm{~h}, 2 \mathrm{~h}, 5 \mathrm{~h}, 1$ day and 6 days. Conjugate concentration for aggregation studies was $1 \mathrm{mkM}$ and $30 \mathrm{nM}$ for UV and fluorescence spectroscopy correspondingly.

\subsection{Synthesis of compounds}

\subsubsection{5,10,15,20-Tetrakis[4-(4,4,5,5-tetramethyl-1,3,2-dioxaborolan-} 2-yl)phenyl]porphyrin (6)

5-[4-(4,4,5,5)-Tetramethyl-1,3,2-dioxaborolan-2-yl)-phenyl]dipyrromethane 4 (prepared according to [34], $174 \mathrm{mg}, 0.5 \mathrm{mmol}$ ) and 4-(4,4,5,5-tetramethyl-1,3,2-dioxaborolan-2-yl)-benzaldehyde 1 (prepared according to [34], $116 \mathrm{mg}, 0.5 \mathrm{mmol}$ ) were dissolved in dry dichloromethane $(50 \mathrm{~mL})$, the resulting solution was degassed under reduced pressure and flushed with argon. Boron trifluoride diethyl etherate complex $(23 \mathrm{mkL}, 0.186 \mathrm{mmol}$ ) was added dropwise and the reaction mixture was stirred at room temperature for $1 \mathrm{~h}$, then DDQ (170 mg, $0.75 \mathrm{mmol}$ ) was added in one portion and the mixture was stirred for additional $1 \mathrm{~h}$. Triethylamine $(26 \mathrm{mkL}, 0.186 \mathrm{mmol})$ was added and the resulting dark solution was filtered through a pad of silica and eluted with dichloromethane to give porphyrin $\mathbf{6}$ as purple solid (105 mg, 38\%). m.p.: $>270{ }^{\circ} \mathrm{C},{ }^{1} \mathrm{H}$ NMR $\left(300 \mathrm{MHz}, \mathrm{CDCl}_{3}\right) \delta: 1.50\left(\mathrm{~s}, 48 \mathrm{H}, \mathrm{CH}_{3}\right), 8.22(\mathrm{~m}$, $16 \mathrm{H}, \mathrm{Ph}-H), 8.82(\mathrm{~s}, 8 \mathrm{H}, \beta$-pyrrole- $H)$.

\subsubsection{5,15-Bis[4-(4,4,5,5-tetramethyl-1,3,2-dioxaborolan-2-} yl)phenyl]-10,20-bispentafluorophenylporphyrin (8)

Product 8 (90 mg, 35\%) was synthesized from 5-[4-(4,4,5,5)tetramethyl-1,3,2-dioxaborolan-2-yl)-phenyl]dipyrromethane 4 (prepared according to [34], $174 \mathrm{mg}, 0.5 \mathrm{mmol}$ ) and pentafluorobenzaldehyde 3 ( $98 \mathrm{mg}, 0.5 \mathrm{mmol}$ ) according to the procedure described for compound 6. m.p.: $>270{ }^{\circ} \mathrm{C} .{ }^{1} \mathrm{H} \mathrm{NMR}\left(300 \mathrm{MHz}, \mathrm{CDCl}_{3}\right)$ $\delta: 1.51\left(\mathrm{~s}, 24 \mathrm{H}, \mathrm{CH}_{3}\right), 8.23(\mathrm{~s}, 8 \mathrm{H}, \mathrm{Ph}-\mathrm{H}), 8.79(\mathrm{~d}, J=5 \mathrm{~Hz}, 4 \mathrm{H}, \beta-\mathrm{pyr}-$ role- $H$ ), 8.94 (d, $J=5 \mathrm{~Hz}, 4 \mathrm{H}, \beta$-pyrrole- $H$ ).

\subsubsection{General procedure for porphyrin deprotection}

Porphyrin $(0.1 \mathrm{mmol})$ was suspended in $4: 1$ mixture of THF/ $\mathrm{H}_{2} \mathrm{O}(7.5 \mathrm{~mL})$ and concentrated $\mathrm{HCl}(2.5 \mathrm{~mL})$ was added. Resulting mixture was stirred for $12 \mathrm{~h}$ at room temperature and red suspension became green. Reaction mixture was diluted with water and precipitate was filtered through fine glass filter and washed several times with water until neutral $\mathrm{pH}$. The resulting green powder was further used without additional purification.

\subsubsection{5,10,15,20-Tetrakis(4-dihydroxylborylphenyl)porphyrin (9)}

Product $\mathbf{9}$ (26 mg, 92\%) was prepared from 5,10,15,20-tetrakis[4(4,4,5,5-tetramethyl-1,3,2-dioxaborolan-2-yl)phenyl]porphyrin 6 (40 mg, $0.036 \mathrm{mmol}$ ). m.p.: >350 ${ }^{\circ} \mathrm{C}$. ${ }^{1} \mathrm{H}$ NMR (300 MHz, $\mathrm{CD}_{3} \mathrm{OD}$ ) $\delta$ : $7.93(\mathrm{~d}, J=7 \mathrm{~Hz}, 8 \mathrm{H}, \mathrm{Ph}-H), 8.14(\mathrm{~d}, J=7 \mathrm{~Hz}, 8 \mathrm{H}, \mathrm{Ph}-H), 8.76(\mathrm{~s}, 8$ $\mathrm{H}, \beta$-pyrrole- $H$ ); ESIMS $m / z$ (rel. int.\%): $805[\mathrm{M}+\mathrm{H}+14]^{+}$(7), 791 $[\mathrm{M}+\mathrm{H}]^{+}(82), 763[\mathrm{M}+\mathrm{H}-28]^{+}(100), 735[\mathrm{M}+\mathrm{H}-56]^{+}(4)$.

\subsubsection{5,15-Bis(4-dioxylborylphenyl)porphyrin (10)}

Product 10 (16 mg, 74\%) was synthesized from 5,15-bis[4(4,4,5,5-tetramethyl-1,3,2-dioxaborolan-2-yl)phenyl]porphyrin 7 (prepared according to [35], $30 \mathrm{mg}, 0.042 \mathrm{mmol}$ ). m.p.: $>350^{\circ} \mathrm{C}$. ${ }^{1} \mathrm{H}$ NMR (300 MHz, CD $\left.{ }_{3} \mathrm{OD}\right) \delta: 8.05$ (d, $\left.J=8 \mathrm{~Hz}, 4 \mathrm{H}, \mathrm{Ph}-H\right), 8.27$ (d, $J=8 \mathrm{~Hz}, 4 \mathrm{H}, \mathrm{Ph}-H$ ), 9.07 (d, $J=5 \mathrm{~Hz}, 4 \mathrm{H}, \beta$-pyrrole- $H$ ), 9.41 (d, $J=5 \mathrm{~Hz}, 4 \mathrm{H}, \beta$-pyrrole- $H$ ), $10.35(\mathrm{~s}, 2 \mathrm{H}$, meso- $H$ ). ESIMS $m / z$ (rel. int.\%): $607[\mathrm{M}+\mathrm{H}+56]^{+}$(5), $579[\mathrm{M}+\mathrm{H}+28]^{+}(14), 565[\mathrm{M}+\mathrm{H}+14]^{+}$ (74), $551[\mathrm{M}+\mathrm{H}]^{+}(100), 523[\mathrm{M}+\mathrm{H}-28]^{+}(40)$. HR ESIMS $m / z$ $551.2063[\mathrm{M}+\mathrm{H}]^{+}$(calcd for $\mathrm{C}_{32} \mathrm{H}_{24} \mathrm{~B}_{2} \mathrm{~N}_{4} \mathrm{O}_{4}+\mathrm{H}, 551.2062$ ).

\subsubsection{5,15-Bis(4-dioxylborylphenyl)-10,20-}

bispentafluorophenylporphyrin (11)

Product 11 (20 mg, 80\%) was prepared from porphyrin $\mathbf{8}$ (30 $\mathrm{mg}$, $0.029 \mathrm{mmol}$ ). m.p.: $>350{ }^{\circ} \mathrm{C} .{ }^{1} \mathrm{H}$ NMR $\left(300 \mathrm{MHz}, \mathrm{CDCl}_{3}\right) \delta: 7.29$ (d, $J=8 \mathrm{~Hz}, 4 \mathrm{H}, \mathrm{Ph}-H), 8.06$ (d, $J=8 \mathrm{~Hz}, 4 \mathrm{H}, \mathrm{Ph}-H), 8.79$ (d, $J=5 \mathrm{~Hz}$, $4 \mathrm{H}, \beta$-pyrrole- $H$ ), 9.00 (d, $J=5 \mathrm{~Hz}, 4 \mathrm{H}, \beta$-pyrrole- $H$ ); ESIMS $m / z$ (rel. int.\%): $896[\mathrm{M}+\mathrm{H}+14]^{+}(4), 883[\mathrm{M}+\mathrm{H}]^{+}(100), 855[\mathrm{M}+\mathrm{H}-28]^{+}$ (36), $827[\mathrm{M}+\mathrm{H}+56]^{+}(10)$. HR ESIMS $m / z 883.1744[\mathrm{M}+\mathrm{H}]^{+}$(calcd for $\mathrm{C}_{44} \mathrm{H}_{22} \mathrm{~B}_{2} \mathrm{~F}_{10} \mathrm{~N}_{4} \mathrm{O}_{4}+\mathrm{H}, 883.1746$ ).

\subsubsection{General procedure for porphyrin-steroid conjugate preparation}

Corresponding porphyrin $(0.025 \mathrm{mmol})$ and steroid (1.1 equiv. per each boronic acid group) were dissolved in methanol (12.5 mM solution). The resulting mixture was stirred at room temperature overnight. Formed purple crystals were filtered and washed with methanol (about $40 \%$ of product was isolated). The filtrate was evaporated under reduced pressure and residue was purified on silica gel (eluent EtOAc-MeOH - 10/1-5/1 for 20E and EtOAc - EtOAc-MeOH (10/1) for EBl) to give purple solid (about $50 \%$ of product).

\subsubsection{Conjugate of 5,10,15,20-tetrakis(4-dihydroxylborylphenyl)por-} phyrin with 20-hydroxyecdysone (12)

Product 12 (31 mg, 80\%) was prepared as a purple solid from porphyrin 9 (12 mg, $0.015 \mathrm{mmol})$ and $20 \mathrm{E}(30 \mathrm{mg}, 0.062 \mathrm{mmol})$. m.p. $(\mathrm{MeOH}):>350{ }^{\circ} \mathrm{C}$. UV $\lambda_{\max }, \mathrm{nm}(\varepsilon, \mathrm{L} /(\mathrm{cm} \cdot \mathrm{mol}))$ : [DMA] 419 (438000), [DMSO] 420 (488000). ${ }^{1} \mathrm{H}$ NMR (500 MHz, $\left.\mathrm{C}_{5} \mathrm{D}_{5} \mathrm{~N}\right) \delta$ : $-2.29(\mathrm{~s}, 2 \mathrm{H}, \mathrm{NH}), 0.72(\mathrm{~s}, 12 \mathrm{H}, \mathrm{C} 18-\mathrm{H}), 0.90(\mathrm{~s}, 12 \mathrm{H}, \mathrm{C} 19-\mathrm{H})$, $1.10(\mathrm{~s}, 24 \mathrm{H}, \mathrm{C} 26 / \mathrm{C} 27-\mathrm{H}), 1.19(\mathrm{~s}, 12 \mathrm{H}, \mathrm{C} 21-\mathrm{H}), 2.99(\mathrm{~m}, 4 \mathrm{H}$, C17-H), 3.09 (d, J=11 Hz, 4H, C5-H), $3.65(\mathrm{~m}, 4 \mathrm{H}, \mathrm{C} 9-\mathrm{H}), 4.24$ (br s, $4 \mathrm{H}, \mathrm{C} 2-\mathrm{H}), 4.31$ (br s, $4 \mathrm{H}, \mathrm{C} 3-\mathrm{H}), 4.64(\mathrm{~d}, J=9 \mathrm{~Hz}, 4 \mathrm{H}, \mathrm{C} 22-$ H), $5.85(\mathrm{~s}, 4 \mathrm{H}, \mathrm{OH}), 6.10(\mathrm{~s}, 4 \mathrm{H}, \mathrm{OH}), 6.19(\mathrm{~s}, 4 \mathrm{H}, \mathrm{OH}), 6.34(\mathrm{~s}, 4 \mathrm{H}$, C7-H), 6.58 (s, 4H, OH), 8.57 (s, 16H, Ph- H), 9.21 (s, 8H, pyrroleH). ${ }^{13} \mathrm{C}$ NMR $\left(125 \mathrm{MHz}, \mathrm{C}_{5} \mathrm{D}_{5} \mathrm{~N}\right) \delta: 18.31,22.04,23.16,24.00$, $25.45,28.10,31.06,31.26,32.59,33.46,35.38,38.96,39.73$, $43.02,48.77,52.41,53.75,69.08,69.15,70.43,85.23,87.26$, $88.43,121.94,122.95,134.89,135.66,146.27,166.46,204.48 . M S$ MALDI-TOF $m / z: 2591[\mathrm{M}+\mathrm{Na}]^{+}(32), 2568[\mathrm{M}+\mathrm{H}]^{+}$(49), 2096 
(49), 2118 (24), 1802 (12), 1680 (33), 1624 (52), 1652 (100), 1207 (42), $1222(14), 1180(32)$.

2.2.9. Conjugate of 5,10,15,20-tetrakis(4-dihydroxylborylphenyl)porphyrin with 24-epibrassinolide (13)

Product 13 (38 mg, 78\%) was obtained as a purple solid from porphyrin 9 (15 mg, $0.019 \mathrm{mmol}$ ) and EBl (prepared according to [36], $40 \mathrm{mg}, 0.083 \mathrm{mmol})$. m.p. (MeOH): $>350{ }^{\circ} \mathrm{C}$. UV $\lambda_{\max }, \mathrm{nm}(\varepsilon$, L/(cm.mol)): [DMA] 420 (312000), [DMSO] 420 (465000). ${ }^{1} \mathrm{H}$ NMR $\left(500 \mathrm{MHz}, \mathrm{C}_{5} \mathrm{D}_{5} \mathrm{~N}\right) \delta:-2.32(\mathrm{~s}, 2 \mathrm{H}, \mathrm{NH}), 0.74(\mathrm{~s}, 12 \mathrm{H}$, C18-H), 0.91 (d, J = 6 Hz, 12H, C-21H), 1.10 (m, 36H, C19-H, C26/ C27-H), 1.20 (d, $J=5 \mathrm{~Hz}, 12 \mathrm{H}, \mathrm{C} 24-\mathrm{H}), 3.67$ (dd, $J=11,4 \mathrm{~Hz}, 4 \mathrm{H}$, $\mathrm{C} 5-\mathrm{H}), 4.01-4.24(\mathrm{~m}, 12 \mathrm{H}, \mathrm{C} 2-\mathrm{H}, \mathrm{C} 7-\mathrm{H}), 4.33(\mathrm{~m}, 4 \mathrm{H}, \mathrm{C} 23-\mathrm{H})$, 4.45 (br.s, 4H, C3-H), 4.68 (d, J=3 Hz, 4H, C22-H), 8.55 (d, $J=6 \mathrm{~Hz}, 8 \mathrm{H}, \mathrm{Ph}-H), 8.61$ (d, $J=6 \mathrm{~Hz}, 8 \mathrm{H}, \mathrm{Ph}-H), 9.14$ (s, 8H, pyrrole- $H$ ). ${ }^{13} \mathrm{C}$ NMR $\left(125 \mathrm{MHz}, \mathrm{C}_{5} \mathrm{D}_{5} \mathrm{~N}\right) \delta: 9.48,11.54,11.91,15.78$, $16.69,21.04,22.39,24.81,27.59,28.07,32.99,38.42,39.57$, $41.59,42.49,42.63,44.84,51.17,52.68,58.25,68.31,68.60$, $70.10,83.07,83.89,120.71,133.80,134.63,145.28,176.51$. MS MALDI-TOF $m / z$ (rel. int.\%): $2591[\mathrm{M}+\mathrm{Na}]^{+}$(3), $2569[\mathrm{M}+\mathrm{H}]^{+}(53)$, 2097 (100), 1624 (35).

\subsubsection{Conjugate of 5,15-bis(4-dioxylborylphenyl)porphyrin with 20-} hydroxyecdysone (14)

Product 14 (35 mg, 93\%) was obtained as a purple solid from porphyrin 10 (14 mg, $0.025 \mathrm{mmol}$ ) and $20 \mathrm{E}(27 \mathrm{mg}, 0.055 \mathrm{mmol})$. m.p. $(\mathrm{MeOH}):>350^{\circ} \mathrm{C}$. UV $\lambda_{\max }, \mathrm{nm}(\varepsilon,(\mathrm{L} / \mathrm{cm} \cdot \mathrm{mol}))$ : [DMA] 407 (335000), [DMSO] 409 (323000). ${ }^{1} \mathrm{H}$ NMR (500 MHz, $\left.\mathrm{C}_{5} \mathrm{D}_{5} \mathrm{~N}\right) \delta$ : -2.65 (s, 2H, NH), 1.17 (s, 6H, C18-H), 1.31 (s, 6H, C19-H), 1.57 $(\mathrm{d}, J=4 \mathrm{~Hz}, 12 \mathrm{H}, \mathrm{C} 26 / \mathrm{C} 27-\mathrm{H}), 1.73(\mathrm{~s}, 6 \mathrm{H}, \mathrm{C}-21 \mathrm{H}), 3.03(\mathrm{t}$, $J=8 \mathrm{~Hz}, 2 \mathrm{H}, \mathrm{C} 17-\mathrm{H}), 3.11(\mathrm{dd}, J=13,2 \mathrm{~Hz}, 2 \mathrm{H}, \mathrm{C} 5-\mathrm{H}), 3.68(\mathrm{~m}$, $2 \mathrm{H}, \mathrm{C} 9-\mathrm{H}), 4.26$ (br d, J = 11 Hz, 2H, C2-H), 4.32 (br s, 2H, C3-H), $4.67(\mathrm{~d}, J=11 \mathrm{~Hz}, 2 \mathrm{H}, \mathrm{C} 22-\mathrm{H}), 6.37(\mathrm{~s}, 2 \mathrm{H}, \mathrm{C} 7-\mathrm{H}), 6.62(\mathrm{~s}, 2 \mathrm{H}$, $\mathrm{OH}), 8.47(\mathrm{~d}, J=8 \mathrm{~Hz}, 4 \mathrm{H}$, pyrrole- $H), 8.60(\mathrm{~d}, J=8 \mathrm{~Hz}, 4 \mathrm{H}$, pyrrole- $H$ ), 9.25 (d, $J=4 \mathrm{~Hz}, 4 \mathrm{H}, \mathrm{Ph}-H), 9.63$ (d, $J=4 \mathrm{~Hz}, 4 \mathrm{H}, \mathrm{Ph}-H)$, $10.62(\mathrm{~s}, 2 \mathrm{H}$, meso- $H) .{ }^{13} \mathrm{C}$ NMR $\left(125 \mathrm{MHz}, \mathrm{C}_{5} \mathrm{D}_{5} \mathrm{~N}\right) \delta: 16.91,20.62$, $21.77,22.62,24.03,26.74,29.67,29.84,31.20,32.06,33.96$, $37.55,38.33,41.62,47.37,51.01,52.36,67.65,67.73,68.98$, $83.80,85.80,86.95,105.79,119.12,121.54,131.00,132.20$, $133.58,134.47,143.95,145.39,147.00,165.08,203.08$. MS MALDI-TOF $m / z$ (rel. int.\%): $1440[\mathrm{M}+\mathrm{H}]^{+}(100), 996$ (60). HR ESIMS $m / z: 1439.7839[\mathrm{M}+\mathrm{H}]^{+}$(calcd for $\mathrm{C}_{86} \mathrm{H}_{104} \mathrm{~B}_{2} \mathrm{~N}_{4} \mathrm{O}_{14}+\mathrm{H}, 1439.7813$ ).

\subsubsection{Conjugate of 5,15-bis(4-dioxylborylphenyl)porphyrin with 24- epibrassinolide (15)}

Product 15 (20 mg, 85\%) was obtained as a purple solid from porphyrin 10 ( $8 \mathrm{mg}, 0.015 \mathrm{mmol}$ ) and EBl (prepared according to [36], $15 \mathrm{mg}, \quad 0.032 \mathrm{mmol})$. m.p. (MeOH): $>350{ }^{\circ} \mathrm{C} .{ }^{1} \mathrm{H} \quad \mathrm{NMR}$ $\left(500 \mathrm{MHz}, \mathrm{C}_{5} \mathrm{D}_{5} \mathrm{~N}\right) \delta:-2.68(\mathrm{~s}, 2 \mathrm{H}, \mathrm{NH}), 0.75(\mathrm{~s}, 6 \mathrm{H}, \mathrm{C} 18-\mathrm{H}), 0.94$ (d, J $=7 \mathrm{~Hz}, 6 \mathrm{H}, \mathrm{C} 21-\mathrm{H}), 1.12(\mathrm{~m}, 18 \mathrm{H}, \mathrm{C} 19-\mathrm{H}, \mathrm{C} 26 / \mathrm{C} 27-\mathrm{H}), 1.26$ $(\mathrm{d}, J=6 \mathrm{~Hz}, 6 \mathrm{H}, \mathrm{C} 24-\mathrm{H}), 3.68$ (dd, $J=12,4 \mathrm{~Hz}, 2 \mathrm{H}, \mathrm{C} 5-\mathrm{H}), 4.04-$ 4.23 (m, 6H, C2-H, C7-H), 4.38 (dd, $J=9,5 \mathrm{~Hz}, 2 \mathrm{H}, \mathrm{C} 23-\mathrm{H}$ ), 4.46 (br.s, 2H, C3-H), 4.71 (d, J=5 Hz, 2H, C22-H), 8.46 (d, J=8 Hz, $4 \mathrm{H}, \mathrm{Ph}-H), 8.64(\mathrm{~d}, J=8 \mathrm{~Hz}, 4 \mathrm{H}, \mathrm{Ph}-H), 9.22$ (d, $J=5 \mathrm{~Hz}, 4 \mathrm{H}$,
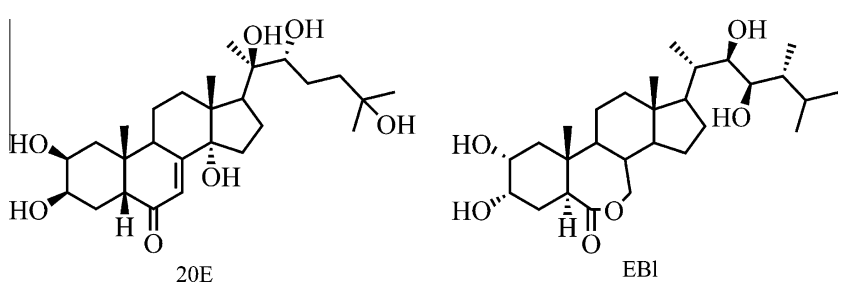

Fig. 1. Chemical structures of 20-hydroxyecdysone (20E) and 24-epibrassinolide $(\mathrm{EBl})$.

pyrrole- $H$ ), 9.62 (d, $J=5 \mathrm{~Hz}, 4 \mathrm{H}$, pyrrole- $H$ ), 10.61 (s, 2H, meso- $H$ ). ${ }^{13} \mathrm{C}$ NMR $\left(125 \mathrm{MHz}, \mathrm{C}_{5} \mathrm{D}_{5} \mathrm{~N}\right) \delta: 9.59,11.59,11.97,15.78,16.87$ $21.06,22.44,24.86,27.72,28.12,29.83,33.00,38.45,39.63$, $41.62,41.68,42.56,42.67,44.86,51.22,52.76,58.30,68.31$, $68.60,70.14,83.17,83.83,106.06,119.30,131.27,132.46,133.98$, $134.81,144.54,145.71,147.27,158.42,176.49$. MS MALDI-TOF $m / z$ (rel. int.\%): $1440[\mathrm{M}+\mathrm{H}]^{+}(41), 1001$ (30), 967 (100). HR ESIMS $m / z: 1439.8561[\mathrm{M}+\mathrm{H}]^{+}$(calcd for $\mathrm{C}_{88} \mathrm{H}_{112} \mathrm{~B}_{2} \mathrm{~N}_{4} \mathrm{O}_{12}+\mathrm{H}, 1439.8541$ ).

\subsubsection{Conjugate of 5,15-bis[4-(4,4,5,5-tetramethyl-1,3,2-}

dioxaborolan-2-yl)phenyl]-10,20-bispentafluorophenylporphyrin with 20-hydroxyecdysone (16)

Conjugate 16 (30 mg, 85\%) was prepared as purple solid from porphyrin $11(17 \mathrm{mg}, 0.020 \mathrm{mmol})$ and $20 \mathrm{E}(21 \mathrm{mg}, 0.044 \mathrm{mmol})$. m.p. $(\mathrm{MeOH}):>350^{\circ} \mathrm{C}$. UV $\lambda_{\max }, \mathrm{nm}(\varepsilon, \mathrm{L} /(\mathrm{cm} \cdot \mathrm{mol})): 417(255000)$ [DMA], 418 (263000) [DMSO]. ${ }^{1} \mathrm{H}$ NMR $\left(500 \mathrm{MHz}, \mathrm{C}_{5} \mathrm{D}_{5} \mathrm{~N}\right) \delta$ : $-2.54(\mathrm{~s}, 2 \mathrm{H}, \mathrm{NH}), 1.16(\mathrm{~s}, 6 \mathrm{H}, \mathrm{C} 18-\mathrm{H}), 1.27$ (s, 6H, C19-H), 1.55 (d, $J=4 \mathrm{~Hz}, 12 \mathrm{H}, \mathrm{C} 26 / \mathrm{C} 27-\mathrm{H}), 1.71(\mathrm{~s}, 6 \mathrm{H}, \mathrm{C} 21-\mathrm{H}), 3.01(\mathrm{t}$, $J=8 \mathrm{~Hz}, 2 \mathrm{H}, \mathrm{C} 17-\mathrm{H}), 3.09$ (dd, $J=13,3 \mathrm{~Hz}, 2 \mathrm{H}, \mathrm{C} 5-\mathrm{H}), 3.66(\mathrm{~m}$, $2 \mathrm{H}, \mathrm{C} 9-\mathrm{H}$ ), 4.25 (br d, J = 12 Hz, 2H, C2-H), 4.31 (br s, 2H, C3-H), $4.65(\mathrm{~d}, J=11 \mathrm{~Hz}, 2 \mathrm{H}, \mathrm{C} 22-\mathrm{H}), 6.34(\mathrm{~s}, 2 \mathrm{H}, \mathrm{C} 7-\mathrm{H}), 6.59$ (s, 2H, $\mathrm{OH}), 8.54$ (d $J=8 \mathrm{~Hz}, 4 \mathrm{H}, \mathrm{Ph}-H), 8.59$ (d, $J=8 \mathrm{~Hz}, 4 \mathrm{H}, \mathrm{Ph}-H), 9.31$ (d, $J=4 \mathrm{~Hz}, 4 \mathrm{H}$, pyrrole- $H), 9.49(\mathrm{~d}, J=4 \mathrm{~Hz}, 4 \mathrm{H}$, pyrrole- $H) .{ }^{13} \mathrm{C}$ NMR $\left(125 \mathrm{MHz}, \mathrm{C}_{5} \mathrm{D}_{5} \mathrm{~N}\right) \delta$ : 18.69, 22.42, 23.56, 24.37, 25.82, $28.48,31.44,31.62,32.98,33.83,35.75,39.35,40.11,43.39$, $49.15,52.78,54.13,69.44,69.51,70.77,85.59,87.66,88.83$, 104.25, 123.33, 123.39, 135.31, 136.01, 145.70, 166.80, 204.83. MS MALDI-TOF $m / z$ (rel. int.\%): $1772[\mathrm{M}+\mathrm{H}](100)$. HR ESIMS $m / z$ : 1771.7524 [M+H] ${ }^{+}$(calcd for $\mathrm{C}_{98} \mathrm{H}_{102} \mathrm{~B}_{2} \mathrm{~F}_{10} \mathrm{~N}_{4} \mathrm{O}_{14}+\mathrm{H}, 1771.7497$ ).

\subsubsection{Conjugate of 5,15-bis[4-(4,4,5,5-tetramethyl-1,3,2-}

dioxaborolan-2-yl)phenyl]-10,20-bispentafluorophenylporphyrin with 24-epibrassinolide (17)

Conjugate 17 (19 mg, 80\%) was prepared as purple solid from porphyrin 11 (12 $\mathrm{mg}, 0.014 \mathrm{mmol}$ ) and EBl (prepared according to [36], $15 \mathrm{mg}, 0.031 \mathrm{mmol})$. m.p. (MeOH): $>350{ }^{\circ} \mathrm{C} .{ }^{1} \mathrm{H}$ NMR $\left(500 \mathrm{MHz}, \mathrm{C}_{5} \mathrm{D}_{5} \mathrm{~N}\right) \delta:-2.68(\mathrm{~s}, 2 \mathrm{H}, \mathrm{NH}), 0.73(\mathrm{~s}, 6 \mathrm{H}, \mathrm{C} 18-\mathrm{H}), 0.90$ (d, $J=7 \mathrm{~Hz}, 6 \mathrm{H}, \mathrm{C} 21-\mathrm{H}), 1.08(\mathrm{~m}, 18 \mathrm{H}, \mathrm{C} 19-\mathrm{H}, \mathrm{C} 26 / \mathrm{C} 27-\mathrm{H}), 1.19$ (d, $J=6 \mathrm{~Hz}, 6 \mathrm{H}, \mathrm{C} 24-\mathrm{H}), 3.67$ (dd, $J=12,4 \mathrm{~Hz}, 2 \mathrm{H}, \mathrm{C} 5-\mathrm{H}), 3.99-$ $4.25(\mathrm{~m}, 6 \mathrm{H}, \mathrm{C} 2-\mathrm{H}, \mathrm{C} 7-\mathrm{H}), 4.33(\mathrm{dd}, J=9,5 \mathrm{~Hz}, 2 \mathrm{H}, \mathrm{C} 23-\mathrm{H}), 4.45$ (br.s, $2 \mathrm{H}, \mathrm{C} 3-\mathrm{H}), 4.68(\mathrm{~d}, J=4.5 \mathrm{~Hz}, 2 \mathrm{H}, \mathrm{C} 22-\mathrm{H}), 8.52(\mathrm{~d}, J=8 \mathrm{~Hz}$, $4 \mathrm{H}, \mathrm{Ph}-H), 8.61(\mathrm{~d}, J=8 \mathrm{~Hz}, 4 \mathrm{H}, \mathrm{Ph}-H), 9.27$ (d, $J=5 \mathrm{~Hz}, 4 \mathrm{H}$, pyrrole- $H$ ), 9.46 (d, $J=5 \mathrm{~Hz}, 4 \mathrm{H}$, pyrrole- $H$ ). MS MALDI-TOF $m / z$ (rel.

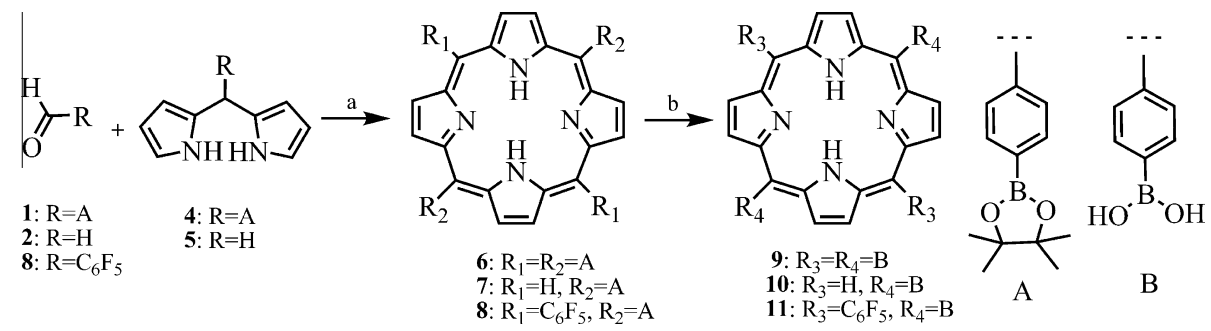

Scheme 1. Reagents and conditions: (a) i: $\mathrm{BF}_{3} \cdot \mathrm{Et}_{2} \mathrm{O}, \mathrm{CH}_{2} \mathrm{Cl}_{2}, 45$ min, r.t., ii: DDQ, 1 h, r.t.; (b) $\mathrm{HCl}_{\text {conc }}, \mathrm{THF}-\mathrm{H}_{2} \mathrm{O}(4: 1)$. 

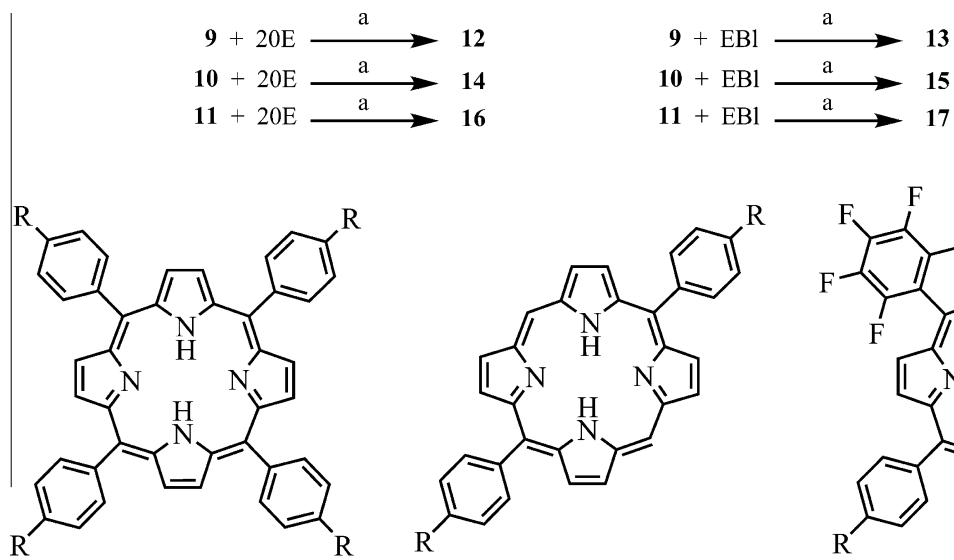<smiles></smiles><smiles>[R]c1ccc(C(c2ccc(C(c3c(F)c(F)c(F)c(F)c3F)=c3ccc(=C(c4ccc([R])cc4)c4ccc[nH]4)[nH]3)[nH]2)=c2ccc(=C(c3ccc([R])cc3)c3c(F)c(F)c(F)c(F)c3F)[nH]2)cc1</smiles>

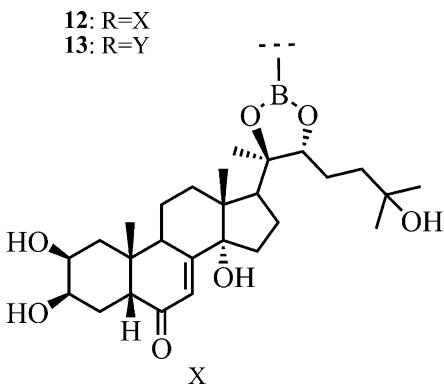

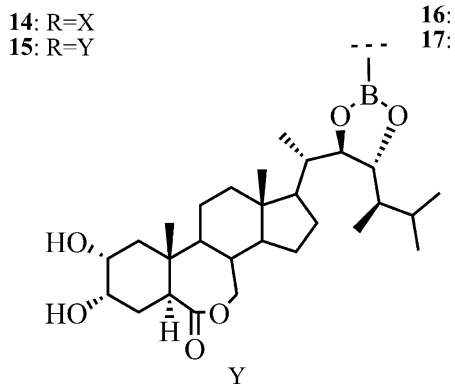

Scheme 2. Reagents and conditions: (a) $\mathrm{MeOH}$, r.t., $12 \mathrm{~h}$
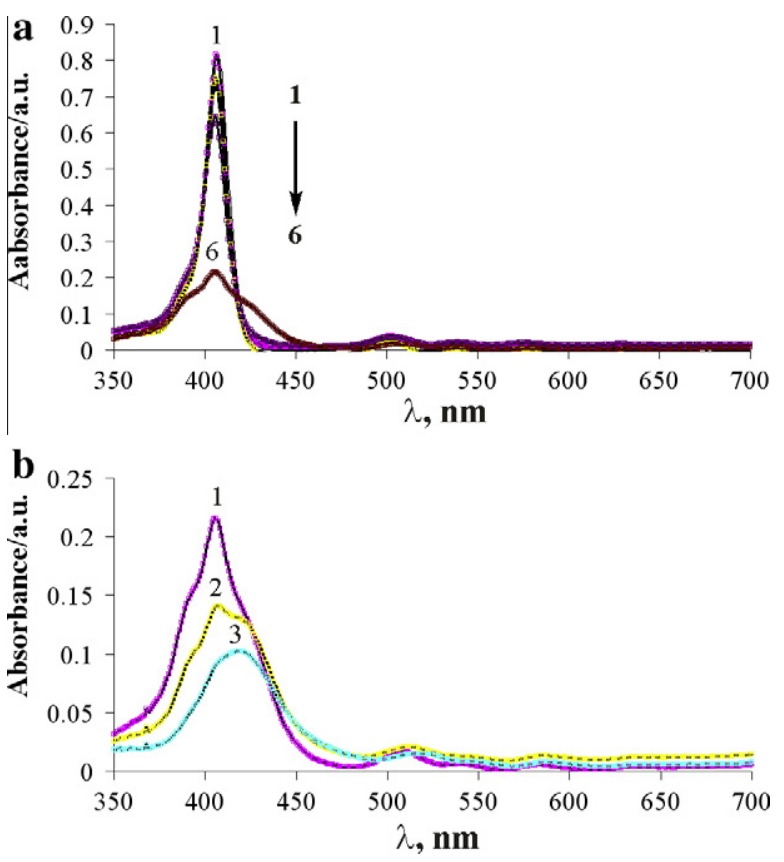

Fig. 2. UV-Vis absorption spectra of conjugate 14 in DMA in aggregation conditions: (a): $(0 \mathrm{~h}) 1-30 \% \mathrm{H}_{2} \mathrm{O}, 2-40 \% \mathrm{H}_{2} \mathrm{O}, 3-50 \% \mathrm{H}_{2} \mathrm{O}, 4-60 \% \mathrm{H}_{2} \mathrm{O}, 5-70 \% \mathrm{H}_{2} \mathrm{O}, 6-$ $80 \% \mathrm{H}_{2} \mathrm{O}$; (b) $\left(80 \% \mathrm{H}_{2} \mathrm{O}\right): 1$ - in $0 \mathrm{~h}, 2$ - in $1 \mathrm{~h}, 3$ - in $5 \mathrm{~h}$.

int.\%): $1772[\mathrm{M}+\mathrm{H}]$ (100), 1299 (65). HR ESIMS m/z: 1771.8252 $[\mathrm{M}+\mathrm{H}]^{+}$(calcd for $\mathrm{C}_{100} \mathrm{H}_{110} \mathrm{~B}_{2} \mathrm{~F}_{10} \mathrm{~N}_{4} \mathrm{O}_{12}+\mathrm{H}, 1771.8225$ ).

\section{Results and discussion}

Out of the numerous oxygenated sterols belonging to ES and BS series, we have chosen 20-hydroxyecdysone (20E) and 24-epibrassinolide (EBl) as ligands for different porphyrin cores (Fig. 1). Both compounds are the most available in the appropriate group of steroids. $20 \mathrm{E}$ is the most abundant among natural ecdysteroids [22], content of which in plants can reach $4 \%$ percent per dry weight. EBl is the first brassinosteroid which is produced for agricultural application [37]. The presence of two diol functions in the molecules of both $20 \mathrm{E}$ and $\mathrm{EBl}$ is a structural feature which is of interest for conjugation purposes. This allowed developing a general strategy for the conjunction process useful both for EBl and 20E.

After having evaluated several possibilities for the conjugation of steroid and porphyrin fragments, we selected those based on the use of boronate esters [38]. Porphyrin-boronic acid derivatives are well known compounds [6,39-42] that can form covalent bonds with 1,2-diols [43-45]. There was an evident concern to manage two diol functions present in the molecules of $20 \mathrm{E}$ and EBl. However, preliminary experience with 24-epibrassinolide methylboronates [46] suggested a possibility to achieve a selective formation of the side-chain functionalized conjugates.

The porphyrins 6-8 were synthesized using Lindsey's method [47] by the reaction of aldehydes 1-3 and dipyrromethanes 4,5 in the presence of a catalytic amount of boron trifluoride-etherate

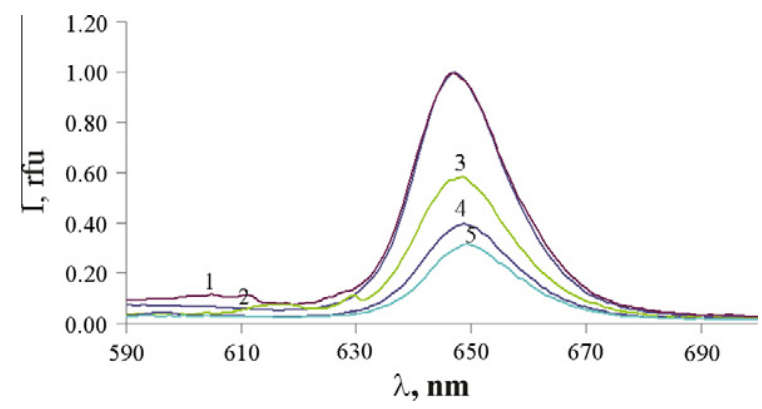

Fig. 3. Fluorescence emission ( $\lambda_{\text {exc }} 420 \mathrm{~nm}$ ) spectra in aggregation conditions in DMSO for compound 12 (measurements directly after water addition): $1-0 \% \mathrm{H}_{2} \mathrm{O}$, $2-20 \% \mathrm{H}_{2} \mathrm{O}, 3-40 \% \mathrm{H}_{2} \mathrm{O}, 4-50 \% \mathrm{H}_{2} \mathrm{O}, 5-60 \% \mathrm{H}_{2} \mathrm{O}$ 
$\mathrm{c}$

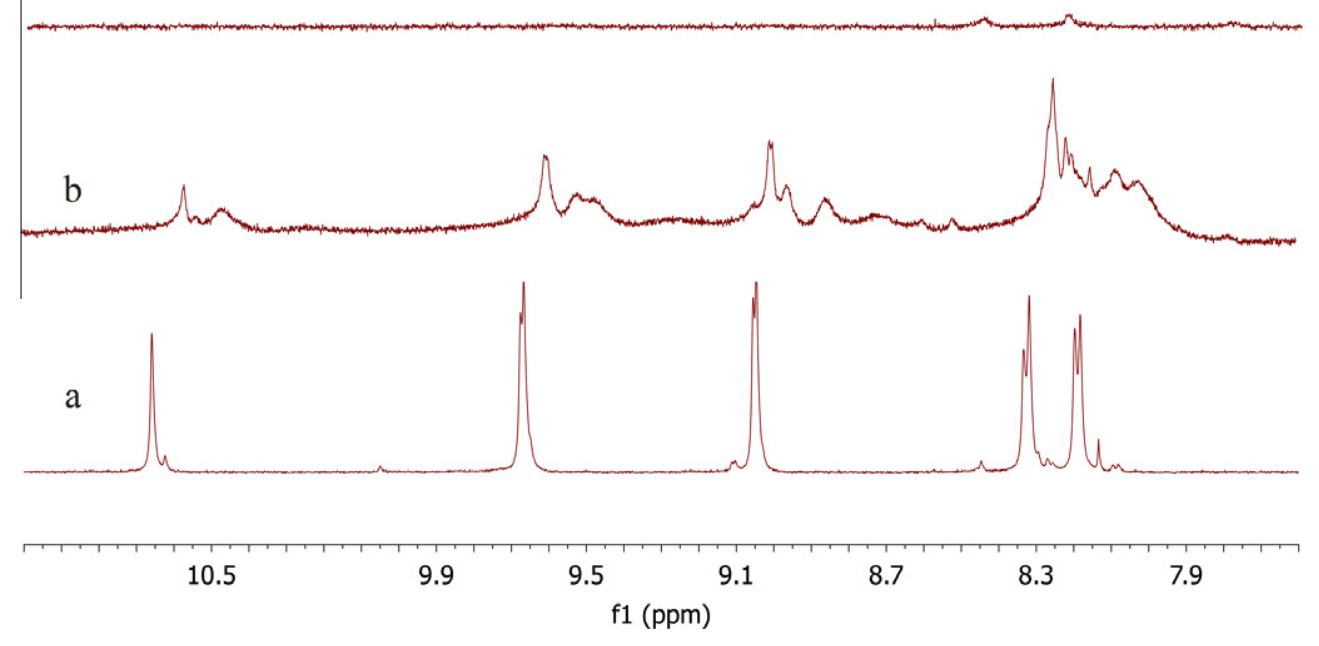

Fig. 4. ${ }^{1} \mathrm{H}$ NMR spectra (aromatic region) of compound 14 in: (a) DMSO- $\mathrm{d}_{6}$; (b) DMSO- $\mathrm{d}_{6}-\mathrm{D}_{2} \mathrm{O}(1: 1)$; (c) DMSO-d $\mathrm{d}_{6}-\mathrm{D}_{2} \mathrm{O}(3: 7)$.

complex with subsequent DDQ oxidation (Scheme 1). Compound 7 was identical in measured parameters to that described in the literature [35].

The removal of the pinacol protecting groups was achieved by treatment of the porphyrins $\mathbf{6 - 8}$ with concentrated $\mathrm{HCl}$ in $\mathrm{THF} /$ water to give the desired unprotected products $\mathbf{9 - 1 1}$ in $\mathbf{7 4 - 9 2 \%}$ yields. Their structures were confirmed by disappearance of the signals corresponding to pinacol methyls in the ${ }^{1} \mathrm{H}$ NMR spectra.

The reaction of the porphyrins 9-11 with slight excess (1.1 eq.) of $20 \mathrm{E}$ and $\mathrm{EBl}$ in methanol gave the corresponding products 12-17 (Scheme 2) resulting from interaction of 20,22-diol group of $20 \mathrm{E}$ or 22,23-diol group of EBl with boronic acid moieties of the porphyrins. All products were isolated as the purple solids in good to excellent yields (78-93\%). These compounds were found to be stable in the solid state as well as in the solution. It is worth mentioning that no conjugates attached to another diol in the cycle A was observed. Evidently that the formation side-chain functionalized conjugates as sole products of this reaction is favored by thermodynamic factors, because 2,3-diol functions in 20E and EBl are less hindered than those in the side chain.

${ }^{1} \mathrm{H}$ NMR spectra of the newly synthesized compounds showed remarkable low-field shifts in the signals of the methyne protons at C-22 in 20E conjugates $\mathbf{1 2 , 1 4}$ and $\mathbf{1 6}$ (from 3.87 to 4.64$4.67 \mathrm{ppm}$ ) and at C-22/C-23 in EBl conjugates 13, 15 and 17 (from 4.04 to $4.68-4.71$ and 3.71 to $4.33-4.38 \mathrm{ppm}$ ).

It's well known that porphyrins and their conjugates could easily form aggregates upon different conditions [48-50]. At room temperature $20 \mathrm{E}$ and $\mathrm{EBl}$ conjugates are well soluble in polar organic solvents such as dimethylsylfoxide (DMSO) and dimethylacetamide (DMA), and they have a sharp Soret band at $\lambda_{\max }=420,407$ or $416 \mathrm{~nm}$ depending on the structure of the porphyrin part. Addition of water to the DMA or DMSO solutions of conjugates resulted in gradual decreasing of the absorption intensity signals which indicates random aggregates' formation. The red shift in UV absorption spectra was observed with time (up to several hours) for all conjugates studied confirming the formation of organized and orientated J-aggregates [51]. A typical change of UV-Vis spectra as a function of water content and time is shown in Fig. 2 for the conjugate 14.

The water-driven aggregation process for steroid-porphyrin conjugates was also confirmed by fluorescence, $\mathrm{CD}$ - and ${ }^{1} \mathrm{H}$ NMR spectra. Thus, fluorescence emission observed in DMSO and DMA for all conjugates decreased with water addition (Fig. 3). In general, absorption and fluorescence properties of $20 \mathrm{E}$ and $\mathrm{EBl}$ conjugates were the same, although 20E conjugates being more polar required higher water content to initiate aggregation (20-30\% for EBl against $40-50 \%$ for $20 \mathrm{E}$ ). Porphyrin part had no significant influence on the spectral characteristics and aggregation properties.

By an example of compound 14, Fig. 4 shows the ${ }^{1} \mathrm{H}$ NMR spectral changes of these conjugates during the aggregation process. It can be seen that by adding $50 \%$ of deuterated water to DMSO- $\mathrm{d}_{6}$ solution of 14, which corresponds to an intermediate state (partial aggregation), the proton signals of porphyrin part show complex picture: additional broadened upfield shifted signals are appeared (Fig. 4b) - monomers, dimers, trimers, etc. might be present in the solution. When $70 \%$ water content was reached (complete aggregation conditions), higher aggregated species were formed. Thus the ${ }^{1} \mathrm{H}$ NMR spectrum (aromatic region) of J-aggregates was not observed because of inhomogeneous broadening of the resonances (Fig. 4c) [52]. It should be pointed out that there were no
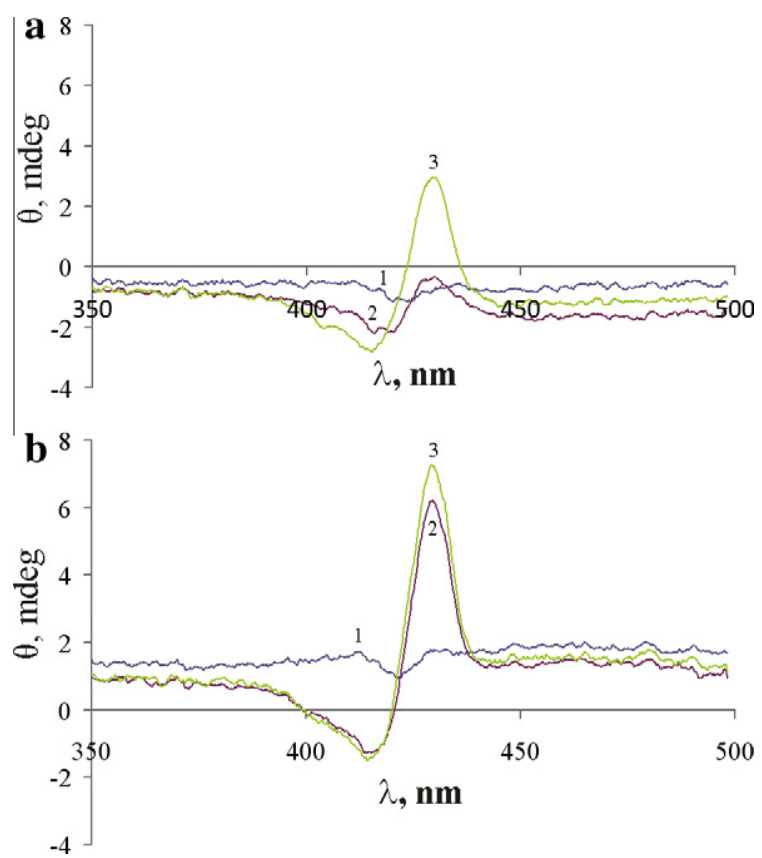

Fig. 5. $\mathrm{CD}$ spectra changes with water content increasing for compound $\mathbf{1 3}$ in: (a) DMA, 1 - 0\% $\mathrm{H}_{2} \mathrm{O}, 2-30 \% \mathrm{H}_{2} \mathrm{O}, 3-40 \% \mathrm{H}_{2} \mathrm{O}$; (b) DMSO, $1-0 \% \mathrm{H}_{2} \mathrm{O}, 2-20 \% \mathrm{H}_{2} \mathrm{O}, 3-$ $40 \% \mathrm{H}_{2} \mathrm{O}$. 
significant changes of steroidal signals. As in previous cases, the same tendency was observed for all conjugates.

Taken as a whole, very similar data were obtained using UVVis-, fluorescence and NMR spectroscopy suggesting the water driven formation of J-aggregates for all the studied compounds. Their CD spectra in pure DMSO and DMA yielded no signals. However, CD measurements of water diluted solutions showed a clear difference between 20E and EBl conjugates. An intense bisignate band with a negative peak at $416 \mathrm{~nm}$, and a positive peak at $430 \mathrm{~nm}$ appeared when water was added to the EBl conjugate solutions both in DMA and DMSO (Fig. 5). At the same time, no changes were observed in CD spectra of $20 \mathrm{E}$ conjugates on water addition. Thereby EBl J-aggregates showed a strong supramolecular chirality whereas $20 \mathrm{E}$ J-aggregates did not. The observed difference may be due to various stacking of the porphyrin macrocyclic rings [53]. Supramolecular chirality in EBl derivatives could originate from the helical edge-to-edge stacked aggregates, and the lack of chirality for $20 \mathrm{E}$ conjugates resulted from linearly or randomly stacking.

\section{Conclusion}

A number of new conjugates of 20-hydroxyecdysone and 24epibrassinolide with three different porphyrins modified by boronic acid residue as a linker were synthesized. Exceptionally high regioselectivity in derivatization of a steroid molecule by porphyrin boronic acids was shown, which resulted in the formation of the side-chain-conjugated boronic esters as sole products. Studies of supramolecular assembly in water-organic solvent systems of the obtained conjugates were carried out by spectral methods. UV-Vis-, fluorescence and NMR spectroscopy confirmed the water driven formation of J-aggregates for all the studied compounds. CD measurements delivered an evidence for the presence of supramolecular chirality in EBl J-aggregates and for its absence in 20E J-aggregates.

\section{Acknowledgments}

This work was supported by by the Ministry of Education, Youth and Sports of CR by Projects No. P304/10/1951, P503/11/ 0616, MSM6046137305, and NATO Grant CBP.EAP.CLG.982972. We thank to Mgr. Martin Svoboda and Mgr. Martina Vermachová, Dept. of Biochemistry and Microbiology, ICT Prague for MALDI spectra measurements.

\section{References}

[1] Ungaro R, Dalcanale E. Supramolecular science. Where it is and Where it is Going. Springer; 1999.

[2] Luliano A, Ruffini A. Effect of the presence of a free hydroxyl group on the enantiodiscrimination properties of cholic acid based CSPs bearing 2naphthylcarbamate and 3,5-dinitrophenylcarbamate moieties in the HPLC resolution of racemic compounds. Tetrahedron Assymmetry 2005;16:38203828.

[3] Sternberg ED, Dolphin D. Porphyrin-based photosensitizers for use in photodynamic therapy. Tetrahedron 1998;54:4151-202.

[4] Koivukorpi J, Sievänen E, Kolehmainen E, Král V. Synthesis, characterization, and saccharide binding studies of bile acid - porphyrin conjugates. Molecules 2007;12:13-24.

[5] Dukh M, Šaman D, Lang K, Pouzar V, Černý I, Drašar P, et al. Steroid-porphyrin conjugate for saccharide sensing in protic media. Org Biomol Chem 2003:1:3458-63.

[6] Hirata O, Kubo Y, Takeuchi M, Shinkai S. Mono- and oligosaccharide sensing by phenylboronic acid-appended 5,15-bis(diarylethynyl)porphyrin complexes. Tetrahedron 2004;60:11211-8.

[7] Hirata O, Yamamoto M, Sugiyasu K, Kubo Y, Ikeda M, Takeuchi M, et al. Allosteric saccharide sensing by a phenylboronic-acids-appended 5,15bis(triarylethynyl) porphyrin. J Supramol Chem 2003;2:133-42.

[8] Kalenius E, Koivukorpi J, Kolehmainen E, Vainiotalo P. Noncovalent saccharide recognition by means of a tetrakis(bile acid)-porphyrin conjugate: selectivity, cooperation, and stability. Eur J Org Chem 2010;6:1052-8.
[9] Bonar-Law RP, Sanders JKM. Polyol recognition by a s teroid-capped porphyrin. enhancement and modulation of misfit guest binding by added water or methanolW. J Am Chem Soc 1995;117:259-71.

[10] Kolehmainen E, Koivukorpi J, Sievänen E, Král V. Novel porphyrin-cholic acid conjugates as receptors for biologically important anions. Supramol Chem 2005;17:437-41.

[11] Virtanen E, Kolehmainen E. Use of bile acids in pharmacological and supramolecular applications. Eur J Org Chem 2004;2004:3385-99.

[12] Tamminen J, Kolehmainen E. Bile acids as building blocks of supramolecular hosts. Molecules 2001;6:21-46.

[13] Huong NTT, Klímková P, Sorrenti A, Mancini G, Drašar P. Synthesis of spiroannulated oligopyrrole macrocycles derived from lithocholic acid Steroids 2009;74:715-20.

[14] Weiss J. Zinc(II)-porphyrin receptors in multi-point molecular recognition: recent progress. J Inclusion Phenom Macrocyclic Chem 2001;40:1-22.

[15] Swamy N, James DA, Mohr SC, Hanson RN, Ray R. An estradiol-porphyrin conjugate selectively localizes into estrogen receptor-positive breast cancer cells. Bioorg Med Chem 2002;10:3237-43.

[16] Bats JW, Haake G, Meier A, Montforts F-P, Scheurich G. Synthesis of oxochlorins from the red blood pigment heme and their transformation into potentially biologically active chlorin derivatives. Liebigs Ann 1995;1995:1617-31.

[17] Shuhua C, Yan C, Hengguang L. Synthesis, configuration and properties of estrogenic steroid-porphyrin enzyme model compounds. Acta Chim Sinica 1997;55:716-22.

[18] Girgenti E, Ricoux R, Mahy J-P. Design and synthesis of a Mn(III)-porphyrin steroid conjugate used as a new cleavable affinity label: on the road to semisynthetic catalytic antibodies. Tetrahedron 2004;60:10049-58.

[19] Vollmer MS, Effenberger F, Stumpfig T, Hartschuh A, Port H, Wolf HC. Steroidbridged anthryloligothienylporphyrins: synthesis and study on the intramolecular energy transfer. J Org Chem 1998;63:5080-7.

[20] Hombrecher HK, Schell C, Thiem J. Synthesis and investigation of a galactopyranosyl-cholesteryloxy substituted porphyrin. Bioorg Med Chem Lett 1996;6:1199-202.

[21] Khipach VA, Zhabinskii VN, de Groot A. Brassinosteroids: a new class of plant hormones. San Diego: Academic Press; 1999.

[22] Smagghe G. Ecdysone: Structures and functions. Springer science + Business, Media BV; 2009.

[23] Zelenka K, Trnka T, Tišlerová I, Monti D, Cinti S, Naitana ML, et al Spectroscopic, morphological, and mechanistic investigation of the solventpromoted aggregation of porphyrins modified in meso-positions by glucosylated steroids. Chem Eur J 2011;17:13743-53.

[24] Dukh M, Drašar P, Černý I, Pouzar V, Shriver JA, Král V et al. Novel deep cavity calix[4]pyrroles derived from steroidal ketones. Supramol Chem 2002; 14.

[25] Monti D, Venanzi M, Gatto E, Mancini G, Sorrenti A. Štěpánek P, et al. Study of the supramolecular chiral assembly of meso-"C-glucoside"-porphyrin derivatives in aqueous media. New J Chem 2008;32:2127-33.

[26] Štěpánek P, Dukh M, Šaman D, Moravcová J, Kniežo L, Monti D, et al. Synthesis and solvent driven self-aggregation studies of meso-"C-glycoside"-porphyrin derivatives. Org Biomol Chem 2007;5:960-70.

[27] Zelenka K, Trnka T, Tišlerová I, Král V, Dukh M, Drašar P. Synthesis of porphyrin receptors modified by glycosylated steroids. Collect Czech Chem Commun 2004;69:1149-60.

[28] Raichyonok TF, Khripach VA, Zhabinskii VN, Konstantinova OV, Drasar PB, Monti D. Synthesis and spectral-luminescence properties of the conjugate of 24-epibrassinolide with porphyrin. J Appl Spectr 2009;76:542-6.

[29] Lettieri R, Monti D, Zelenka K, Trnka T, Drašar P, Venanzi M. Glucosylated steroid-porphyrins as new tools for nanotechnology applications. New J Chem 2012:36:1246-54.

[30] Štěpánek P, Šimák O, Nováková Z, Wimmer Z, Drašar P. Asymmetrically substituted calix[4]pyrrole with chiral substituents. Org Biomol Chem 2011;9:682-3.

[31] Khripach VA, Zhabinskii VN, Litvinovskaya RP. Immunoassays of brassinosteroids. In: Hayat S, Ahmad A, editors. Brassinosteroids: a class of plant hormone. Dordrecht: Springer; 2011. p. 375-92.

[32] Rumlerová-Lipšová A, Barek J, Drašar P, Zelenka K, Pecková K. Polarographic and voltammetric detrmination of meso-tetrakis(4-sulphonatophenyl)porphyrin tetrasodium salt at mercury electrodes. Int J Electrochem Sci 2007;2:235-47.

[33] Strohalm M, Kavan D, Novák P, Volný M, Havliček V. MMass 3: a crossplatform software environment for precise analysis of mass spectrometric data. Anal Chem 2010;82:4648-51.

[34] Yu L, Lindsey JS. Investigation of two rational routes for preparing pphenylene-linked porphyrin trimers. Tetrahedron 2001;57:9285-98.

[35] Senge MO, Shaker YM, Pintea M, Ryppa C, Hatscher SS, Ryan A, et al. Synthesis of meso-substituted ABCD-type porphyrins by functionalization reactions. Eur J Org Chem 2010;2010:237-58.

[36] Khripach VA, Zhabinskii VN, Olkhovik VK, Ivanova GI, Zhernosek EV, Kotyatkina AI. Improved synthesis of epibrassinolide. Russ J Org Chem 1994;30:1735-40.

[37] Khripach V, Zhabinskii V, de Groot A. Twenty years of brassinosteroids: steroidal plant hormones warrant better crops for the XXI century. Ann Bot 2000;86:441-7.

[38] Hall DG, editor. Boronic Acids: Preparation and Applications in Organic Synthesis and Medicine. Weinheim: WILEY-VCH Verlag; 2005.

[39] Imada T, Kijima H, Takeuchi M, Shinkai S. Selective binding of glucose-6phosphate, 3,4-dihydroxyphenylalanine (DOPA) and their analogs with a boronic-acid-appended metalloporphyrin. Tetrahedron 1996;52:2817-26. 
[40] Sugasaki A, Ikeda M, Takeuchi M, Shinkai S. Novel oligosaccharide binding to $\mathrm{Ce}^{\mathrm{iv}}$ bis(porphyrinate) double decker: effective amplification of a binding signal through positive homotropic allosterism. Angew Chem, Int Ed 2000;39:3839-42.

[41] Sugasaki A, Sugiyasu K, Ikeda M, Takeuchi M, Shinkai S. First successful molecular design of an artificial lewis oligosaccharide binding system utilizing positive homotropic allosterism. J Am Chem Soc 2001;123:10239-44.

[42] Zhang C, Suslick KS. Syntheses of boronic-acid-appended metalloporphyrins as potential colorimetric sensors for sugars and carbohydrates. J Porphyrins Phthalocyanines 2005;9:659-66.

[43] Mader HS, Wolfbeis OS. Boronic acid based probes for microdetermination of saccharides and glycosylated biomolecules. Microchim Acta 2008:162:1-34.

[44] Jin S, Cheng Y, Reid S, Li M, Wang B. Carbohydrate recognition by boronolectins, smallmolecules, and lectins. Med Res Rev 2010;30:171-257.

[45] Hargrove AE, Reyes RN, Riddington I, Anslyn EV, Sessler JL. Boronic acid porphyrin receptor for ginsenoside sensing. Org Lett 2010;12:4804-7.

[46] Khripach VA, Zhabinskii VN, Zhiburtovich YY, Ivanova GV, Konstantinova OV Tsavlovskii DV, et al. Preparation and synthetic application of partially protected brassinosteroids. Steroids 2010;75:27-33.
[47] Ravikanth M, Strachan J-P, Li F, Lindsey JS. Trans-substituted porphyrin building blocks bearing iodo and ethynyl groups for applications in bioorganic and materials chemistry. Tetrahedron 1998;54:7721-34.

[48] Zhang Y, Wu Y. Different J-type aggregates of meso-tetrakis (4hydroxyphenyl) porphyrin $\left(\mathrm{H}_{2} \mathrm{THPP}\right)$ formed in different solvents. Chin Chem Lett 2005;16:534.

[49] White WI. In: Dolphin D, editor. The Porphyrins. New York: Academic Press; 1978

[50] Würthner F, Kaiser TE, Saha-Möller CR. J-Aggregates: from serendipitous discovery to supramolecular engineering of functional dye materials. Angew Chem Int Ed 2011;50:3376-410.

[51] Mishra A, Behera RK, Behera PK, Mishra BK, Behera GB. Cyanines during the 1990s: a review. Chem Rev 2000;100:1973-2011.

[52] Ma HL, Wu JJ, Liang WJ, Chao JB. Study on the association phenomenon of cyclodextrin to porphyrin J-aggregates by NMR spectroscopy. J Inclusion Phenom Macrocyclic Chem 2007;58:221-6.

[53] Liu L, Li Y, Liu M. Acidichromism and supramolecular chirality of tetrakis(4sulfonatophenyl)porphyrin in organized molecular films. J Phys Chem (C) 2008;112:4861-6. 